\title{
Research Paper: The Interrater and Intrarater Reliability of the Preterm Infant Oral Feeding Readiness Assessment Scale
}

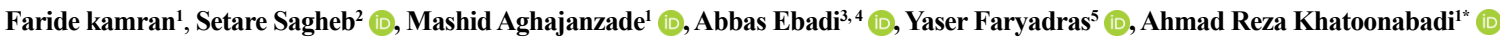 \\ 1. Department of Speech Therapy, School of Rehabilitation, Tehran University of Medical Sciences, Tehran, Iran. \\ 2. Department of Pediatrics, School of Medicine, Shariati Hospital, Tehran University of Medical Sciences, Tehran, Iran. \\ 3. Behavioral Sciences Research Center, Lifestyle Institute, Baqiyatallah University of Medical Sciences, Tehran, Iran. \\ 4. Department Nursing, Faculty of Nursing, Baqiyatallah University of Medical Sciences, Tehran, Iran. \\ 5. Department of Foreign Languages, Faculty of Literature and Humanities, Kharazmi University, Tehran, Iran.
}

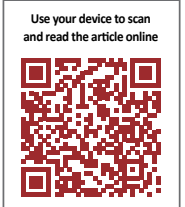

Citation: Kamran F, Sagheb S, Aghajanzade M, Ebadi A, Faryadras Y, Khatoonabadi A. The Interrater and Intrarater Reliability of the Preterm Infant Oral Feeding Readiness Assessment Scale. Journal of Modern Rehabilitation. 2019; 13(1):31-38. http://dx.doi.org/10.32598/JMR.13.1.31

http://dx.doi.org/10.32598/JMR.13.1.31

Article info:

Received: 10 Jun 2018

Accepted: 13 Sep 2018

Available Online: 01 Jan 2019

Keywords:

Premature infant, Feeding behavior, Assessment

\begin{abstract}
A B S T RACT
Introduction: One of the most challenging decisions is to assess the preterm infant's transition from tube feeding to oral feeding. Thus, we require a reliable tool for determining the time to start oral feeding. This study aimed to measure the interrater and intrarater reliability of the Preterm Infant Oral Feeding Readiness Assessment scale (PIOFRA).

Materials and Methods: This study was an observational, cross-sectional study. The study participants were preterm infants who had been hospitalized in the Neonatal Intensive Care Unit of Shariati Hospital affiliated to Tehran University of Medical Sciences, between December 2017 and February 2018. The inclusion criteria were absence of neurological and gastrointestinal disorders or major congenital anomalies, Apgar score 3 or more in the first 1 minute, and 5 or more in the first 5 minutes. The exclusion criteria included family's unwillingness to participate in the study, infant's death, or a sudden change that affects neonates' nutritional status, like cerebral hemorrhage or intestinal problems.
\end{abstract}

Results: The interrater and intrarater reliability of the total PIOFRA scale was good Intraclass Correlation Coefficients (ICC $>0.75$ ). The interrater and intrarater reliability of most items were good and excellent, with weighted kappa more than 0.50 , with the exception of lip posture and especially stress sign, with weighted kappa less than 0.40 .

Conclusion: Generally, most items of the PIOFRA scale had acceptable interrater and intrarater reliability. Also, the interrater and intrarater reliabilities of the total POFRAs score was good.

* Corresponding Author:

Ahmad Reza Khatoonabadi, PhD.

Address: Department of Speech Therapy, School of Rehabilitation, Tehran University of Medical Sciences, Tehran, Iran.

Tel: +98 (21) 77684879

E-mail: a.rkhatoonabadi@gmail.com 


\section{Introduction}

A

chieving an efficient feeding skill is one of the biggest challenges of preterm infants [1]. The integration of maturation, physiological stability, behavioral state organization, and coordinated sucking-swallowing-breathing are prerequisites of successful feeding. In other words, successful feeding indicates neurobehavioral maturation. Furthermore, it is a prerequisite of sufficient oral nutrient intake [2].

The majority of healthy full-term infants experience safe oral feeding because they can coordinate sucking, swallowing, and breathing. However, preterm infants do not show these maturities, and they will achieve these skills gradually [1]. Therefore, preterm infants, before achieving independent oral feeding need a period of enteral feeding. One of the most challenging decisions is to when stop tube feeding and start oral feeding. Besides physiological stability and consistent weight gain, this independent oral feeding is another factor necessary for the baby before discharge from the hospital.

Delays in discharge increase financial costs on the families and government $[1,3]$. One of the objectives of the speech-language pathologist is to facilitate the transition to independent oral feeding [4]. Accordingly, care services focus not only on survival of preterm infants but also on comprehensive, humanized, and preventive care, within an individualized and developmental care perspective, which requires training the neonatal care team [5].

Clinically, recognizing the exact time to start oral feeding in stable infants is difficult. Besides, the evaluation is based on isolated factors such as gestational age, postmenstrual age, weight, and scheduled feeding regimens that focus on the volume of milk that consumes the infant [3]. The majority of health professionals, in this process, consider isolated data for the infants, without assessing oral motor skills, general conditions, and neuro-psychomotor development $[4,5]$. These parameters for safely initiating oral feeding are not sufficient [4].

Several scales are available for clinicians to evaluate the infant's feeding skills [6]. Some tools of infant's feeding skills assessment such as LATCH (L: Latches; A: Audible swallowing; T: Nipple type; C: Level of comfort; H: Holding infant) were developed for infants who are fed from the breast [7]. The early feeding skills assessment for preterm infants, the support of oral feeding for fragile infants, and the infant driven feeding assessment were designed for infants who started oral feeding [8-10].

Neonatal Oral Motor Assessment Scale (NOMAS) is one of the earliest tools that assesses an infant's oral motor function and sucking. The NOMAS is a 28 -item assessment tool that evaluates the infant's jaw and tongue movements and classifies an infant's sucking patterns as normal, disorganized, or dysfunctional. The NOMAS is used from birth through eight weeks' corrected age. However, it only assesses the movements of the tongue and jaw $[11,12]$. Thus, clinicians need a tool to measure all aspects of oral skills and to gain the necessary information whether the infant needs intervention before initiation of oral feeding.

The only tool that assesses all dimensions of oral-motor skills in preterm infants before feeding and, more importantly, helps to recognize oral-motor problems of infants before the start of oral feeding is Preterm Infant Oral Feeding Readiness Assessment scale (PIOFRA). The PIOFRA scale is used to assess preterm infant oral feeding readiness. It was designed by Fujinaga et al. in Brazil. The aspects of this scale includes corrected age, behavioral organization (behavioral state, global posture, and tonus), oral posture (lips and tongue), oral reflexes (rooting, sucking, biting, and gag) and nonnutritive sucking (tongue movement, tongue cupping, jaw movement, sucking strain, sucking and pause, maintenance of rhythm of sucking and pause, maintenance of state of alertness and stress signs). A score ranging from 0 to 2 was attributed to each item of the protocol. The performance of the infant is determined by the sum of the scores obtained, which can vary from 0 to 36 . The validity and reliability of this scale were determined in 2007 and 2013. The scale has a sensitivity of $60 \%$ and a $75 \%$ specificity $[4,5,12,13]$.

Accordingly, the assessment of nonnutritive sucking with other dimensions of the infant's global behavior, such as gestational age, posture, and global tonus and behavioral state, are among signs which demonstrate maturity for the transition to oral feeding [5].

Therefore, it would be helpful to use a reliable scale that provides useful clinical guidelines for speech-language pathologists to assess infant's oral feeding readiness skills comprehensively. Because there is not a reliable scale to assess preterm infant oral feeding readiness in speech therapy clinics in Iran and no research has ever tested the reliability of the PIOFRA scale in Iran, we aimed to measure the interrater and intrarater reliability of this scale. 


\section{Materials and Methods}

This study was an observational, cross-sectional study conducted on preterm infants 34 weeks old or younger who had been hospitalized in the Neonatal Intensive Care Unit of Shariati Hospital affiliated to Tehran University of Medical Sciences, between December 2017 and February 2018.

The infants' parents gave their consent before the study. The study objectives were written in the consent forms. Moreover, some points were explained to all in- fant's parents. Preterm infants that had clinical stability were entered in the sampling that was the convenience sampling method.

The sample consisted of 30 preterm infants; 17 females and 13 males. Their Mean \pm SD gestational age was $223.96 \pm 11.73$ days. Their Mean \pm SD weight at birth was $1642 \pm 320.54 \mathrm{~g}$ About $90 \%$ of the samples experienced respiratory problems at birth. Table 1 presents the clinical data for infants of this research. The inclusion criteria were infants who lacked any neurological and gastrointestinal disorders or major congenital anoma-

Table 1. The infants' clinical data

\begin{tabular}{|c|c|c|c|c|c|c|}
\hline Infants & $\begin{array}{l}\text { Gestational Age } \\
\text { (d) }\end{array}$ & $\begin{array}{c}\text { Birth } \\
\text { Weight (g) }\end{array}$ & $\begin{array}{c}\text { Sex } \\
\text { (Female: 1, } \\
\text { Male: 2) }\end{array}$ & $\begin{array}{l}\text { 1-Min Apgar } \\
\text { Score }\end{array}$ & $\begin{array}{l}\text { 5-Min Apgar } \\
\text { Score }\end{array}$ & Problems at Birth \\
\hline 1 & 230 & 1705 & 2 & 8 & 9 & Respiratory discomfort \\
\hline 2 & 210 & 1370 & 1 & 5 & 8 & Respiratory discomfort \\
\hline 3 & 224 & 1930 & 2 & 4 & 7 & Respiratory discomfort \\
\hline 4 & 212 & 1650 & 1 & 3 & 8 & Respiratory discomfort \\
\hline 5 & 237 & 1710 & 1 & 5 & 8 & Respiratory discomfort \\
\hline 6 & 238 & 1630 & 2 & 4 & 6 & Respiratory discomfort \\
\hline 7 & 238 & 1790 & 2 & 8 & 9 & Respiratory discomfort \\
\hline 8 & 238 & 2290 & 2 & 8 & 9 & None \\
\hline 9 & 226 & 1460 & 1 & 8 & 9 & Respiratory discomfort \\
\hline 10 & 210 & 1960 & 1 & 8 & 9 & Respiratory discomfort \\
\hline 11 & 231 & 1935 & 1 & 7 & 8 & Respiratory discomfort \\
\hline 12 & 224 & 1170 & 2 & 8 & 9 & Respiratory discomfort \\
\hline 13 & 238 & 2110 & 1 & 8 & 9 & None \\
\hline 14 & 216 & 1670 & 1 & 3 & 8 & Respiratory discomfort \\
\hline 15 & 224 & 1375 & 1 & 8 & 9 & Respiratory discomfort \\
\hline 16 & 219 & 1500 & 1 & 7 & 9 & Respiratory discomfort \\
\hline 17 & 231 & 2060 & 2 & 8 & 10 & Respiratory discomfort \\
\hline 18 & 224 & 1810 & 1 & 8 & 9 & Respiratory discomfort \\
\hline 19 & 224 & 1170 & 2 & 8 & 9 & Respiratory discomfort \\
\hline 20 & 238 & 1205 & 1 & 4 & 8 & Respiratory discomfort \\
\hline 21 & 217 & 1605 & 2 & 7 & 9 & Respiratory discomfort \\
\hline 22 & 211 & 1730 & 2 & 3 & 8 & Respiratory discomfort \\
\hline 23 & 210 & 1400 & 1 & 5 & 8 & Respiratory discomfort \\
\hline 24 & 223 & 1905 & 1 & 6 & 7 & Respiratory discomfort \\
\hline 25 & 196 & 1000 & 2 & 4 & 7 & Respiratory discomfort \\
\hline 26 & 238 & 1330 & 1 & 8 & 9 & Respiratory discomfort \\
\hline 27 & 230 & 1810 & 2 & 8 & 9 & Respiratory discomfort \\
\hline 28 & 238 & 2110 & 1 & 6 & 8 & None \\
\hline 29 & 221 & 1540 & 2 & 8 & 9 & Respiratory discomfort \\
\hline 30 & 203 & 1330 & 1 & 4 & 6 & Respiratory discomfort \\
\hline
\end{tabular}


lies, and their Apgar score was three or more in the first minute and five or more in the first five minutes of birth. The exclusion criteria included family's unwillingness to participate in the study, infant's death, or a sudden change that affects neonates' nutritional status, like cerebral hemorrhage or intestinal problems.

To test the interrater reliability of the scale, two speechlanguage pathologists assessed each infant 15 minutes before their feeding time. The observers had no verbal contact. Therapists accomplished the assessment concurrently for each infant, and they did not talk to each other. The first therapist awakened the infant using the tactile, visual, and auditory stimuli. This therapist handled the infant to assess the behavioral organization, oral posture, and presence of oral rooting and vomiting reflexes. Both therapists noticed these behaviors concurrently. Each of therapists evaluated the biting and sucking reflexes and nonnutritive sucking (during one minute) separately by the gloved pinkie.

To examine intrarater reliability, one of the therapists once assessed each infant and recorded the infant's behaviors on video during the first assessment. After one week, this therapist observed the video that was recorded during the first assessment and scored the infant's oral performance again.

\section{Statistical analysis}

All data were analyzed in SPSS V. 23 The interrater and intrarater reliabilities of the PIOFRA scale were determined by Intraclass Correlation Coefficients (ICC) based on the total score. The coefficient less than 0.5 indicates poor reliability; the coefficient between 0.51 and 0.75 represents moderate reliability, and the coefficient greater than 0.75 represents good reliability [14]. Both interrater and intrarater agreement for ordinal scaled data were examined by weighted kappa. Evaluation criteria for kappa, using guidelines described in Cicchetti and Sparrow (1981) and Fleiss (1981) were as follows: Fair: 0.40 to 0.59 ; Good: 0.60 to 0.74 ; and Excellent: $>0.74$ $[15,16]$.

\section{Results}

Tables 2 and 3 present the results of the weighted kappa for each item. The interrater reliability demonstrated excellent agreement for $9(30 \%)$ items, good for $6(20 \%)$ items, fair for $1(3.3 \%)$ item, and unsatisfactory agreement for $1(3.3 \%)$ item (Table 2). The intrarater reliability indicated excellent agreement for $8(26.6 \%)$ items, good for $5(20 \%)$ items, fair for $2(3.3 \%)$ items, and unsatisfactory agreement for $2(6.6 \%)$ items (Table 3$)$.

The ICC and Standard Error of Measurement (SEM) of total Preterm Infant Oral Feeding Readiness Assessment (PIOFRA) scale score are presented in Tables 4 and 5. The interrater reliability of the total PIOFRA scale score was good (ICC: 0.84 ; 95\% CI: 0.66-0.92) (Table 4). The intrarater reliability of the total PIORAF scale score was good, too (ICC: 0.97 ; 95\% CI: 0.940.98) (Table 5).

\section{Discussion}

The majority of the protocols of preterm infant feeding assessment are designed to determine the exact time for starting oral feeding [17]. The recognition of the best time for initiating oral feeding help infant to experience satisfaction from oral feeding after being wean from tube feeding [18]. Furthermore, it contributes to reducing the duration of achieving full oral feeding, decreasing the length of hospitalization, and the financial costs for the family and government $[1,2]$. Moreover, an exact assessment helps the therapists to determine what interventions infant needs for achieving full oral feeding [19].

PIOFRA scale is one of the instruments that can be used easily, quickly, and without any harm, for infants to help speech-language pathologists in their clinical assessments. Also, the scale considers the variety of dimensions such as level of maturity, behavioral organization, state of consciousness, and oral-motor skills [5, 13]. This study aimed to investigate interrater and intrarater reliabilities of the PIOFRA scale. The results of this study demonstrated that the interrater and intrarater reliabilities of most items of this scale were good and excellent, with weighted kappa more than 0.60 . However, lip posture, tongue posture, and especially stress sign were exception, because their weighted kappa was less than 0.52 . Also, the interrater and intrarater reliabilities of the total PIOFRA scale score was good, with ICC greater than 0.75 .

There is not any research that tests the reliability of the PIOFRA scale except the Fujinaga et al. study. The results of their study demonstrated that the interrater reliability of the majority of the items were excellent and good except tongue cupping, maintenance of sucking, and stress sign [5]. Stress sign was the only unsatisfactory item that was the same between Fujinaga et al. and our study. One of the reasons for the low level of interobserver agreement is the alteration of infant's clinical 
Table 2. The interrater reliability of PIOFRA scale for each item

\begin{tabular}{|c|c|c|}
\hline Scale Item & Kappa & Qualitative Assessment \\
\hline Behavioral state & 0.84 & Excellent \\
\hline Global posture & 0.63 & Good \\
\hline Global tune & 1.00 & Excellent \\
\hline Lip posture & 0.50 & Fair \\
\hline Tongue posture & 0.76 & Excellent \\
\hline Rooting reflex & 0.64 & Good \\
\hline Sucking reflex & 0.78 & Excellent \\
\hline Biting reflex & 0.82 & Excellent \\
\hline Gag reflex & 0.71 & Good \\
\hline Tongue movement & 0.62 & Good \\
\hline Tongue cupping & 0.73 & Good \\
\hline Jaw movement & 0.68 & Good \\
\hline Sucking strain & 0.84 & Excellent \\
\hline Sucking and pause & 0.85 & Excellent \\
\hline Maintenance of sucking/pause & 0.77 & Excellent \\
\hline Maintenance of alert state & 0.95 & Excellent \\
\hline Stress sign & 0.35 & Unsatisfactory \\
\hline
\end{tabular}

Table 3. The intrarater reliability of PIOFRA scale for each item

\begin{tabular}{|c|c|c|}
\hline Scale Item & Карра & Qualitative Assessment \\
\hline Behavioral state & 0.90 & Excellent \\
\hline Global posture & 0.68 & Good \\
\hline Global tune & 0.72 & Good \\
\hline Lip posture & 0.38 & Unsatisfactory \\
\hline Tongue posture & 0.51 & Fair \\
\hline Rooting reflex & 0.74 & Good \\
\hline Sucking reflex & 0.90 & Excellent \\
\hline Biting reflex & 0.65 & Good \\
\hline Gag reflex & 0.67 & Good \\
\hline Tongue movement & 0.89 & Excellent \\
\hline Tongue cupping & 0.60 & Good \\
\hline Jaw movement & 0.84 & Excellent \\
\hline Sucking strain & 0.90 & Excellent \\
\hline Sucking and pause & 0.88 & Excellent \\
\hline Maintenance of sucking/pause & 0.94 & Excellent \\
\hline Maintenance of alert state & 0.95 & Excellent \\
\hline Stress sign & 0.09 & Unsatisfactory \\
\hline
\end{tabular}


Table 4. The interrater reliability of the total PIOFRA scale score

\begin{tabular}{ccccc}
\hline Total Score & Mean \pm SD & ICC & 95\% Cl & SEM \\
\hline PIOFRA scale & $19.56 \pm 7.74$ & 0.84 & $0.66-0.92$ & 3.09 \\
\hline
\end{tabular}

$J \mathrm{MR}$

Table 5. The intrarater reliability of the total PIOFRAscore

\begin{tabular}{ccccc}
\hline Total Score & Mean \pm SD & ICC & $95 \%$ Cl & SEM \\
\hline PIOFRA scale & $20.70 \pm 7.941$ & 0.97 & $0.94-0.98$ & 1.35 \\
\hline
\end{tabular}

$J \mathrm{MR}$

stability. Infant's manipulation is the reason to change infant's stability $[20,21]$.

Infant's lip posture and tongue posture in every infant is different in various states [21]. Fujinaga et al. indicated a high level of interobserver agreement while our research demonstrated lower level of agreement, especially in lip posture compared to that in the study by Fujinaga and associates. This difference is probably because of using observers with different experiences and precision in each study. The weighted kappa of the behavioral state, global posture, gag reflex, and maintenance of alert state in interrater and intrarater reliabilities of the present research was nearly similar to Fujinaga et al. study findings.

Tongue cupping refers to when sides of the tongue elevate, and a groove is created in the middle of the tongue, and the number of sucks and pause refers to maintenance of sucking and pause rhythm [5, 22]. There is a significant difference in the tongue cupping and maintenance of sucking items between the findings of Fujinaga et al. study and our research. Although the interrater reliability of these items in our study was good and excellent, in the study of Fujinaga et al., they were unsatisfactory. These differences may be due to the difficulty of measurements because the assessment of the PIOFRA scale is subjective and both items rely on tactile sensitivity and exact observation. Moreover, the infant's behavioral state may influence sucking patterns [21].

The reliability finding of this research and results of Bolzan et al. study illustrated that the accuracy of the PIOFRA scale is moderate; however, the concordance the oral feeding skill level and the PIOFRA scale was weak; thus, another assessment tool should be used besides PIOFRA scale in clinical assessments [4].

One of the biggest problems of the PIOFRA scale is the evaluation of oral function subjectively. Therefore, it is necessary to study the reliability of this scale in comparison with an objective examination, for instance, ultrasound imaging and electromyography. Using objective methods, muscular activity can be observed during nonnutritive sucking, for example, tongue elevation during nonnutritive sucking and also tongue and hyoid movements during nutritive sucking are observable by ultrasound imaging $[23,24]$.

\section{Conclusion}

Generally, most items of the PIOFRA scale demonstrated excellent and good interrater and intrarater reliabilities, except for stress sign and lip posture. Also, the interrater and intrarater reliabilities of the total PIOFRA scale score was good. We suggest that the next studies examine the reliability of this scale by two professional feeding therapists or the result of the PIOFRA scale be compared with objective examination.

\section{Ethical Considerations}

\section{Compliance with ethical guidelines}

The Institutional Review Board, School of Rehabilitation, and the Ethics Committee of Teheran University of Medical Sciences approved this study protocol (IR. TUMS.FNM.REC.1397.010). The consent form was given to the infants' parents before participating in their study. The study objectives and interventions have been written in the contest form. Moreover, those will be explained to the infants' parents.

\section{Funding}

The present paper was extracted from the MSc. thesis of the first author, Faride Kamran, Department of Speech Therapy, School of Rehabilitation, Tehran University of Medical Sciences; under the sponsorship of the Deputy for Education, Tehran University of Medical Sciences. 


\section{Authors contributions}

All authors contributed equally in preparing this article

\section{Conflict of interest}

All authors declare no conflicts of interest.

\section{Acknowledgements}

We thank all nurses and parents who collaborated with us in the neonatal intensive care unit and the neonatal ward of Shariati Hospital.

\section{References}

[1] Crowe L, Chang A, Wallace K. Instruments for assessing readiness to commence suck feeds in preterm infants: Effects on time to establish full oral feeding and duration of hospitalisation. Cochrane Database of Systematic Reviews. 2016 (8):CD005586. [DOI:10.1002/14651858.CD005586.pub3]

[2] Howe TH, Lin KC, Fu CP, Su CT, Hsieh CL. A review of psychometric properties of feeding assessment tools used in neonates. Journal of Obstetric, Gynecologic \& Neonatal Nursing. 2008; 37(3):338-49. [DOI:10.1111/j.1552-6909.2008.00240.x] [PMID]

[3] Giannì ML, Sannino P, Bezze E, Plevani L, Esposito C, Muscolo S, et al. Usefulness of the Infant Driven scale in the early identification of preterm infants at risk for delayed oral feeding independency. Early Human Development. 2017; 115:18-22. [DOI:10.1016/j.earlhumdev.2017.08.008] [PMID]

[4] de Paula Bolzan G, Berwig LC, Prade LS, Cuti LK, de Carvalho Yamamoto RC, da Silva AMT, et al. Assessment for oral feeding in preterm infants. CoDAS. 2016; 28(3):284-8. [DOI:10.1590/2317-1782/20162015115]

[5] Fujinaga CI, Zamberlan NE, de Oliveira Rodarte MD, Scoch CGS. Reliability of an instrument to assess the readiness of preterm infants for oral feeding. Pró-Fono Revista de Atualização Científica. 2007; 19(2):143-50. [DOI:10.1590/S010456872007000200002] [PMID]

[6] Pados B, Park J, Estrem H, Awotwi A. Assessment tools for evaluation of oral feeding in infants younger than 6 months. Advances in Neonatal Care. 2016; 16(2):143-50. [DOI:10.1097/ ANC.0000000000000255] [PMID] [PMCID]

[7] Jensen D, Wallace S, Kelsay P. LATCH: A breastfeeding charting system and documentation tool. Journal of Obstetric, Gynecologic, \& Neonatal Nursing. 1994; 23(1):27-32. [DOI:10.1111/j.1552-6909.1994.tb01847.x] [PMID]

[8] Thoyre SM, Shaker CS, Pridham KF. The early feeding skills assessment for preterm infants. Neonatal Network. 2005; 24(3):7. [DOI:10.1891/0730-0832.24.3.7] [PMID] [PMCID]

[9] Philbin M, Ross E. The SOFFI reference guides: Text, algorithms, and appendices: A manualized method for quality bottle feedings. The Journal of Perinatal \& Neonatal Nurs- ing. 2011; 25(4):360-80. [DOI:10.1097/JPN.0b013e31823529da] [PMID] [PMCID]

[10] Ludwig SM, Waitzman KA. Changing feeding documentation to reflect infant-driven feeding practice. Newborn and Infant Nursing Reviews. 2007; 7(3):155-60. [DOI:10.1053/j. nainr.2007.06.007]

[11] Da Costa SP, Van Der Schans CP. The reliability of the Neonatal Oral-Motor Assessment scale. Acta Paediatrica. 2008 97(1):21-6. [DOI:10.1111/j.1651-2227.2007.00577.x] [PMID]

[12] Thoyre SM, Pados BF, Shaker CS, Fuller K, Park J. Psychometric properties of the early feeding skills assessment tool. Advances in Neonatal Care. 2018; 18(5):E13-E23. [DOI:10.1097/ANC.0000000000000537] [PMID]

[13] Fujinaga CI, de Moraes SA, Zamberlan-Amorim NE, Castral TC, de Almeida e Silva A, Scochi CGS. Clinical validation of the preterm oral feeding readiness assessment scale. Revista Latino-Americana de Enfermagem. 2013; 21(Spec):140-5. [DOI:10.1590/S0104-11692013000700018] [PMID]

[14] Portney LG, Watkins MP. Foundations of clinical research: Applications to practice. Upper Saddle River, New Jersi: Pearson/Prentice Hall; 2009.

[15] Cicchetti DV, Sparrow SA. Developing criteria for establishing interrater reliability of specific items: Applications to assessment of adaptive behavior. American Journal of Mental Deficiency. 1981; 86(2):127-37. [PMID]

[16] Fleiss JL, Levin B, Paik MC. The measurement of interrater agreement. In: Fleiss JL, editor. Statistical methods for rates and proportions. New York: Wiley, John and Sons; 1981.

[17] Eichenwald EC, Blackwell M, Lloyd JS, Tran T, Wilker $\mathrm{RE}$, Richardson DK. Inter-neonatal intensive care unit variation in discharge timing: Influence of apnea and feeding management. Pediatrics. 2001; 108(4):928-33. [DOI:10.1542/ peds.108.4.928] [PMID]

[18] Neiva FC, Leone CR, Leone C, Siqueira LL, Uema KA Evangelista D, et al. Non-nutritive sucking evaluation in preterm newborns and the start of oral feeding: A multicenter study. Clinics. 2014; 69(6):393-7. [DOI:10.6061/clinics/2014(06)05]

[19] Bauer MA, de Carvalho Yamamoto RC, Weinmann ARM, Keske-Soares M. [Evaluation of sensory-motor-oral stimulation in the transition from gastric tube to full oral feeding in preterm newborns (Portuguese)]. Revista Brasileira de Saúde Materno Infantil. 2009; 9(4):429-34. [DOI:10.1590/S151938292009000400007]

[20] Thoyre SM. Developmental transition from gavage to oral feeding in the preterm infant. Annual Review of Nursing Research. 2003; 21(1):61-94. [DOI:10.1891/0739-6686.21.1.61] [PMID]

[21] Fujinaga CI, Maltauro S, Stadler ST, Cheffer ER, Aguiar $\mathrm{S}$, Amorin NEZ, et al. Behavioral state and the premature's readiness performance to begin oral feeding. Revista CEFAC 2018; 20(1):95-100. [DOI:10.1590/1982-021620182015317]

[22] Fujinaga CI. [Preterm readiness for oral feeding: Reliability and clinical validation of an evaluation instrument (Portuguese)] [PhD. dissertation]. Ribeirão Preto: Universidade de São Paulo; 2005. [DOI:10.11606/T.22.2005.tde-25052007171954] 
[23] Miller JL, Kang SM. Preliminary ultrasound observation of lingual movement patterns during nutritive versus nonnutritive sucking in a premature infant. Dysphagia. 2007; 22(2):150-60. [DOI:10.1007/s00455-006-9058-z] [PMID]

[24] Gomes CF, Thomson Z, Cardoso JR. Utilization of surface electromyography during the feeding of term and preterm infants: A literature review. Developmental Medicine \& Child Neurology. 2009; 51(12):936-42. [DOI:10.1111/j.14698749.2009.03526.x] [PMID] 